\section{Atlas of Nuclear Cardiology}

V. Dilsizian and J. Narula, Eds.

New York, NY: Springer, 2013, 457 pages, \$209

Nuclear cardiology imaging is solidly based on many branches of science and engineering, and the strengths of nuclear techniques are related to physiologic, biochemical, and molecular properties. The ability to define myocardial perfusion, viability, and ventricular function from a single study has become a powerful diagnostic and prognostic tool, and thus nuclear cardiology is now recognized as a distinct clinical entity. Nuclear cardiology procedures have become the cornerstone of the decision-making process to appropriately select patients for medical or interventional therapy, as well as to monitor the effectiveness of that therapy. Innovative imaging strategies in nuclear cardiology will propel the field into molecular imaging and personalized medicine.

The object of this fourth edition of the Atlas of Nuclear Cardiology is to elucidate the role of cardiovascular nuclear procedures in the clinical practice of cardiology. By allowing quantification of blood flow, PET has led to a better understanding of the physiologic mechanism underlying cardiovascular diseases. Targeted molecular imaging and image-guided therapy further improve the management of heart diseases. In this atlas, diagnostic algorithms and schematic diagrams integrated with nuclear cardiology procedures are generally interspersed with superb color figures and tables to emphasize key concepts in cardiovascular physiology, pathology, metabolism, and innervation. References are updated, and the index is convenient.
The atlas consists of 12 chapters written by 27 international experts. The first chapter presents the principles of nuclear cardiology imaging along with instrumentation and image acquisition. Chapters 2-4 detail SPECT and PET radiopharmaceuticals, imaging techniques, and stressors for the detection of coronary artery disease. Chapter 5 deals with the benefits of quantification of myocardial perfusion, and chapter 6 reviews techniques for radionuclide angiography and myocardial perfusion SPECT. Chapter 7 discusses myocardial perfusion imaging for risk stratification and for identifying survival benefits with medical therapy versus revascularization. Chapters 8 and 9 focus on cardiac metabolism and neurohumoral targets for preventing heart failure. Chapter 10 addresses cardiac imaging for the diagnosis and risk stratification of patients with acute coronary syndromes. Chapters 11 and 12 examine radionuclide techniques for myocardial innervation and molecular imaging of atherosclerosis.

I am sure that this atlas will serve as a foundation for clinicians and a reference guide for scientists within and outside the field of nuclear cardiology. I highly recommend this book to trainees; to practitioners of nuclear medicine, diagnostic radiology, cardiology, and family medicine; and to those interested in imaging science.

\section{E. Edmund Kim}

University of California at Irvine

101 The City Dr. S.

Orange, $C A 92868$

E-mail: edmundek@uci.edu

Published online $\mathbf{m}$

DOI: 10.2967/jnumed.113.132191 